\title{
Interactions between surfactants and
}

\section{\{1,4-phenylene-[9,9-bis(4-phenoxy-butylsulfonate)]fluorene-2,7-diyl $\}$}

\author{
H.D. Burrows ${ }^{\text {a,* }}$, V.M.M. Lobo ${ }^{\text {a }}$, J. Pina ${ }^{\text {a }}$, M.L. Ramos ${ }^{\text {a }}$, J. Seixas de Melo ${ }^{\text {a }}$, \\ A.J.M. Valente ${ }^{\mathrm{a}}$, M.J. Tapia ${ }^{\mathrm{b}}$, S. Pradhan ${ }^{\mathrm{c}}$, U. Scherf ${ }^{\mathrm{c}}$, S.I. Hintschich ${ }^{\mathrm{d}}$, \\ C. Rothe ${ }^{d}$, A.P. Monkman ${ }^{\mathrm{d}}$ \\ a Departamento de Química, Universidade de Coimbra, 3004-535 Coimbra, Portugal \\ ${ }^{\mathrm{b}}$ Departamento de Química, Universidad de Burgos, Plaza Misael Bañuelos s/n, Burgos 09001, Spain \\ ${ }^{c}$ Macromolecular Chemistry and Institute for Polymer Technology, Bergische Universität Wuppertal, \\ D-42097 Wuppertal, Germany \\ d Department of Physics, University of Durham, Durham DH1 3LE, UK
}

Available online 5 July 2005

\begin{abstract}
The interaction between the water-soluble anionic conjugated copolymer poly \{1,4-phenylene-[9,9-bis(4-phenoxy-butylsulfonate)]fluorene2,7-diyl (PBS-PFP) and various surfactants has been studied in aqueous solution by UV-vis absorption spectra, fluorescence and electrical conductivity. It is suggested from the linear dependence of absorbance, fluorescence and electrical conductivity on concentration that in the absence of surfactant, moderately stable dispersions are formed. These are affected in different ways on adding cationic, anionic or neutral surfactants. With the cationic cetyltrimethylammonium bromide, quenching of fluorescence intensity and lifetime, and formation of a new emission occurs at concentrations well below the critical micelle concentration $(\mathrm{cmc})$. Electrical conductivity measurements indicate a discontinuity at surfactant/polymer ratio corresponding to electroneutrality, due to complexation. With the anionic sodium dodecyl sulfate, fluorescence quenching is also observed, but is attributed to formation of some mixed polymer/surfactant aggregate. The most striking changes are observed with the non-ionic pentaethyleneglycol monododecyl ether $\left(\mathrm{C}_{12} \mathrm{E}_{5}\right)$, where a blue shift in fluorescence emission, dramatic increases in lifetime and quantum yield, and changes in electrical around the cmc are interpreted in terms of incorporation of single polymer chains in elongated cylindrical micelles. This is supported by ${ }^{1} \mathrm{H}$ NMR spectroscopic measurements.
\end{abstract}

(C) 2005 Elsevier B.V. All rights reserved.

Keywords: Polymer-surfectant interactions; Fluorene copolymers; Conjugated polyelectrolytes

\section{Introduction}

Conjugated organic polymers are technologically important materials with applications in areas such as light emitting diodes, organic lasers, photovoltaic systems and thin film transistors [1,2]. Particular emphasis has focused on polymers and copolymers of 9,9-substituted fluorenes, since these emit light in the blue with high efficiencies and also show fairly high stability [3,4]. In addition, they behave as fairly rigid "hairy-rods" [5] and also order into liquid crystalline structures [6].

\footnotetext{
* Corresponding author. Tel.: +351 239854482; fax: +351 239827703

E-mail address: burrows@ci.uc.pt (H.D. Burrows).
}

Recently, interest has been shown in water-soluble ionic derivatives of these and related conjugated polymers, which show potential in areas such as chemical and biological sensors [7-14] and self-assembly systems [15-18]. In addition, with the introduction of ink jet printing for fabricating light emitting diode (LED) displays and other organic electronic systems, the demand for water-based conjugated polymer systems is likely to increase [19]. The water-soluble conjugated organic polymers function as polyelectrolytes, and show interesting interactions with surfactants $[9,10,13]$. In particular, with the anionic polymer, poly(2,5-methoxy-propyloxysulfonate phenylene vinylene) (MPS-PPV), the fluorescence quantum yield is enhanced by more than an order of magnitude on adding the cationic 


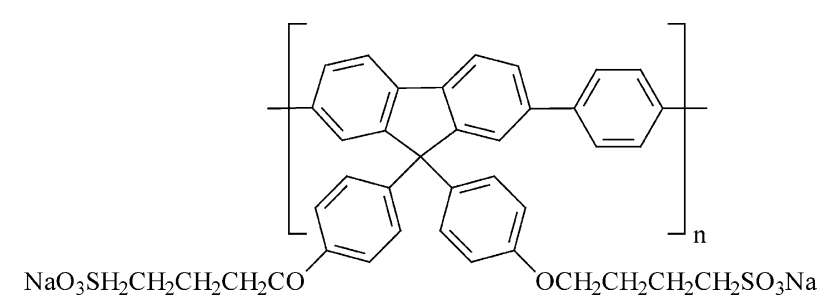

Fig. 1. Structure of PBS-PFP.

surfactant dodecyltrimethylammonium bromide, although there is no significant change in the emission spectrum [9]. In contrast, with a neutral, water-soluble sugar substituted poly(p-phenyleneethynylene), marked changes in emission spectra were observed on addition of various surfactants, which the authors termed surfactochromicity [13]. We have observed that with aqueous solutions of the watersoluble copolymer poly $\{1,4$-phenylene-[9,9-bis(4-phenoxybutylsulfonate)]fluorene-2,7-diyl (PBS-PFP, Fig. 1), a blue shift in fluorescence spectrum together with an increase in both fluorescence quantum yield and lifetime is observed on adding the nonionic surfactant pentaethyleneglycol monododecyl ether $\left(\mathrm{C}_{12} \mathrm{E}_{5}\right)$ [20]. This occurs at surfactant concentrations close to the critical micelle concentration (cmc) of the surfactant, and is attributed to the break-up of PBS-PFP aggregates, and incorporation of the polymer into cylindrical micelles. With these rigid rod polyfluorene derivatives, it is likely that the polymer-surfactant interactions will be somewhat different from those in more flexible polymers, where a variety of conformational changes are possible, and where association is commonly treated with a "pearl-necklace model" [21]. We present a more general study on the interaction of the anionic PBSPFP with surfactants in aqueous solutions, considering its behaviour with the cationic cetyltrimethylammonium bromide (CTAB, $\mathrm{CH}_{3}\left(\mathrm{CH}_{2}\right)_{15} \mathrm{~N}\left(\mathrm{CH}_{3}\right)_{3} \mathrm{Br}$ ), the anionic sodium dodecyl sulfate (SDS, $\mathrm{CH}_{3}\left(\mathrm{CH}_{2}\right)_{11} \mathrm{OSO}_{3} \mathrm{Na}$ ) and the neutral $\mathrm{C}_{12} \mathrm{E}_{5}$.

\section{Experimental}

\subsection{Materials}

The polymer PBS-PFP $\left(\bar{M}_{\mathrm{n}}=\sim 6500 \mathrm{~g} \mathrm{~mol}^{-1}\right)$ was synthesised by condensation of 2,7-dibromo-9,9-bis(4-sulfonylbutoxyphenyl)fluorene (A) and 1,4-phenylenediboronic acid using $\mathrm{Pd}\left(\mathrm{PPh}_{3}\right)_{4}$ as catalyst. Full details of synthesis and characterisation have been given elsewhere [20,22]. Cetyltrimethylammonium bromide (CTAB, Aldrich), sodium dodecyl sulfate (SDS, Aldrich), pentaethylene glycol monododecyl ether $\left(\mathrm{C}_{12} \mathrm{E}_{5}\right.$, Fluka) and other reagents were of the purest grade available, and were used without further treatment. All solutions were prepared using Millipore-Q water.

\subsection{Apparatus and methods}

\subsubsection{Spectroscopic measurements}

Absorption spectral measurements were made in $1 \mathrm{~cm}$ quartz cuvettes on a Shimadzu UV-2100 spectrophotometer. The steady-state fluorescence spectra were measured with a Fluorolog 3-22 instrument with $0.5 \mathrm{~nm}$ excitation and emission bandwidths. Fluorescence decays were measured using a home-built Time-Correlated Single Photon Counting apparatus with an $\mathrm{N}_{2}$ filled IBH 5000 coaxial flashlamp or an IBH NanoLED $\left(\lambda_{\text {exc }}=373 \mathrm{~nm}\right)$ as excitation source. This was coupled to a Jobin-Ivon monochromator, Philips XP2020Q photomultiplier, and Canberra instruments timeto-amplitude converter and Multichannel Analyser. Alternate measurements ( 1000 counts per cycle), controlled by Decay ${ }^{\circledR}$ software (Biodinâmica-Portugal), of the pulse profile at 337, 356 or $373 \mathrm{~nm}$ and the sample emission were performed until 1 to $2 \times 10^{4}$ counts at the maximum were reached.[23] The fluorescence decays were analysed using the modulating functions method of Striker with automatic correction for the photomultiplier "wavelength shift"[24]. All experiments were carried out at room temperature.

Solution electrical resistances were measured with a Wayne-Kerr model 4265 Automatic LCR meter at $1 \mathrm{kHz}$. A Shedlovsky-type conductance cell was used [25]. The cell constant (approximately $0.8465 \mathrm{~cm}^{-1}$ ) was determined to $\pm 0.02 \%$ from measurements with $\mathrm{KCl}$ (reagent grade, recrystallised and dried using the procedure and data from [26]. Measurements were made at $25.00 \pm 0.01{ }^{\circ} \mathrm{C}$ in a Grant thermostat bath.

${ }^{1} \mathrm{H}$ NMR spectra of PBS-PFP were run on solutions in $\mathrm{D}_{2} \mathrm{O}$ on a Varian Unity $500 \mathrm{MHz}$ spectrometer.

\subsection{Sample preparation}

As indicated in Section 3.1, dispersions of PBS-PFP in water are not stable over extended periods of time. However, at a PBS-PFP concentration around $6 \times 10^{-3} \mathrm{~g} \mathrm{dm}^{-3}$ it was possible to prepare systems which were stable enough for fluorescence and electrical conductivity measurements by keeping under continuous stirring overnight and making the relevant measurements immediately afterwards.

\section{Results and discussion}

\subsection{Behaviour of PBS-PFP in aqueous solution}

The polymer PBS-PFP did not dissolve readily in water. However, by stirring overnight it was possible to obtain a relatively stable dispersion, although on leaving a solid precipitated out of this. With freshly prepared solutions, a relatively broad absorption was observed, with a maximum at $381 \mathrm{~nm}$ (Fig. 2). The absorbance of this increased linearly with polymer concentration, in agreement with the Beer-Lambert law. Similarly, a linear increase in fluorescence with concentration 


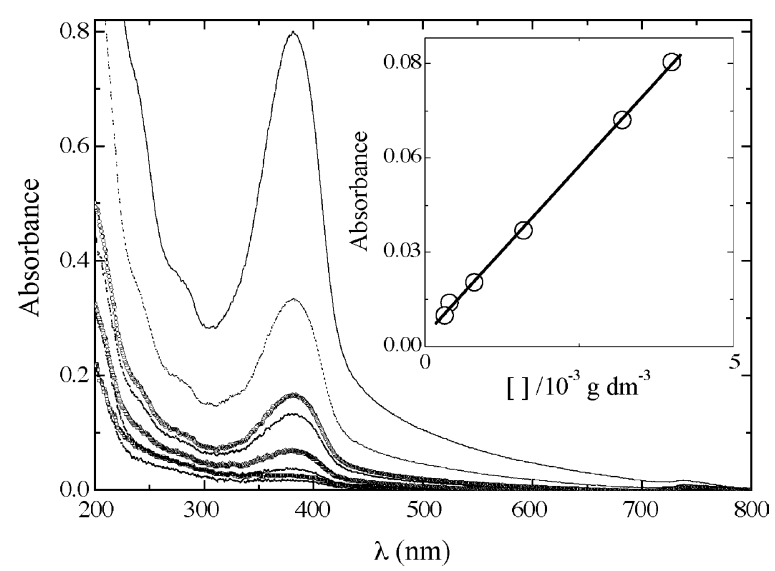

Fig. 2. Absorption spectra of PBS-PFP in water at various concentrations. Inset: Beer-Lambert plot of absorption at $381 \mathrm{~nm}$ as function of polymer concentration.

was observed. The precipitation probably results from association of PBS-PFP aggregates in solution, which have previously been reported for $p$-phenylene and fluorene based polymers $[4,20,27,28]$, and other conjugated polyelectrolytes [29]. The absorbance behaviour will be further discussed with electrical conductivity results in Section 3.3.

\subsection{Fluorescence spectral and lifetime measurements}

Solutions of PBS-PFP in water showed a structured fluorescence between 400 and $550 \mathrm{~nm}$, which is similar to that observed with other phenyl-fluorene copolymers [29]. Upon addition of various concentrations of CTAB, a decrease in intensity of the fluorescence was observed and was accompanied by formation of a new emission around $525 \mathrm{~nm}$. There were no changes in the emission spectra of the dominant species (Fig. 3(a)). This contrasts with the behaviour of MPSPPV with cationic surfactants, where a dramatic increase in fluorescence intensity was observed. These differences pos-
Table 1

Fluorescence lifetimes observed for aqueous solutions of PBS-PFP upon addition of $\mathrm{CTAB}$

\begin{tabular}{lll}
\hline [CTAB] $\left(\mathrm{mol} \mathrm{dm}^{-3}\right)$ & \multicolumn{2}{l}{ Fluorescence lifetime $(\mathrm{ns})$} \\
\cline { 2 - 3 } & Observed at $420 \mathrm{~nm}$ & Observed at $530 \mathrm{~nm}$ \\
\hline 0 & 0.36 & - \\
$3.3 \times 10^{-7}$ & 0.25 & - \\
$6.7 \times 10^{-7}$ & 0.24 & - \\
$7.9 \times 10^{-6}$ & 0.22 & - \\
$3.1 \times 10^{-5}$ & 0.27 & $0.20^{\mathrm{a}}$ \\
& & 1.49 \\
$2.0 \times 10^{-4}$ & 0.29 & $0.19^{\mathrm{a}}$ \\
& & 1.68 \\
\hline
\end{tabular}

${ }^{a}$ Biexponential decay.

sibly arise from the relatively rigid-rod structure of PBS-PFP, compared with the random coil MPS-PPV.

As with other polymer-surfactant systems [21], all these changes occur at surfactant concentrations (ca. $4 \times 10^{-6} \mathrm{~mol} \mathrm{dm}^{-3}$ ) very much lower than the cmc of CTAB $\left(8 \times 10^{-4} \mathrm{~mol} \mathrm{dm}^{-3}[30]\right)$. The results strongly suggest complexation between the oppositely charged surfactant and polymer favoured by electrostatic interactions. However, because of the rigid structure of PBS-PFP, we feel that the polymer-surfactant aggregates have a somewhat different structure from the pearl-necklace structure seen in more flexible polymer-surfactant systems. The origin of the new band at $525 \mathrm{~nm}$ is discussed in detail elsewhere [22]. These changes were accompanied by a decrease in the fluorescence lifetime observed at the main band and appearance of a second component with rather longer lifetime at $525 \mathrm{~nm}$ (Table 1).

In contrast, on addition of the anionic SDS to aqueous solutions of PBS-PFP, although quenching of fluorescence occurred (Fig. 3(b)), no new bands were observed, and there was no observable change in the fluorescence lifetime of the polymer within the time resolution of our system. Again, emission maxima did not shift on addition of the surfactant.
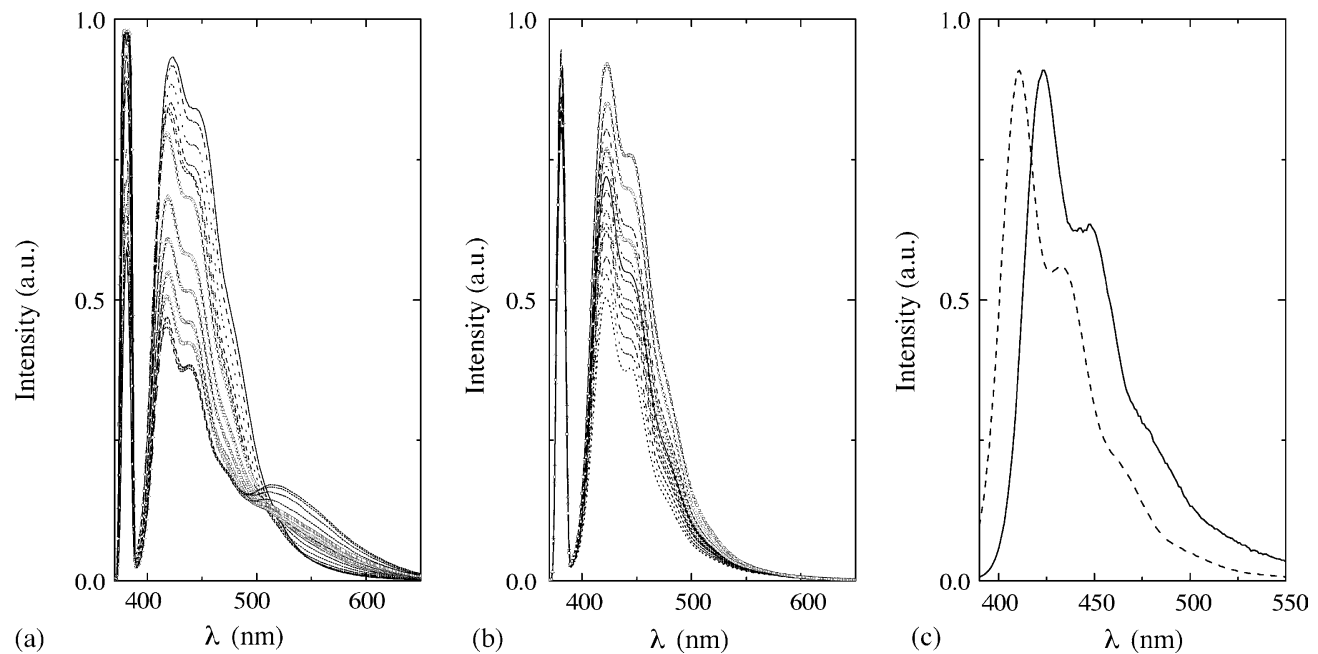

Fig. 3. Fluorescence spectra of aqueous solutions of PBS-PFP upon addition of: (a) CTAB; (b) SDS; (c) $\mathrm{C}_{12} \mathrm{E}_{5}$. Note blue shift in fluorescence spectra in (c) on adding surfactant. In (a) and (b) the Rayleigh scattering peak is also shown. 


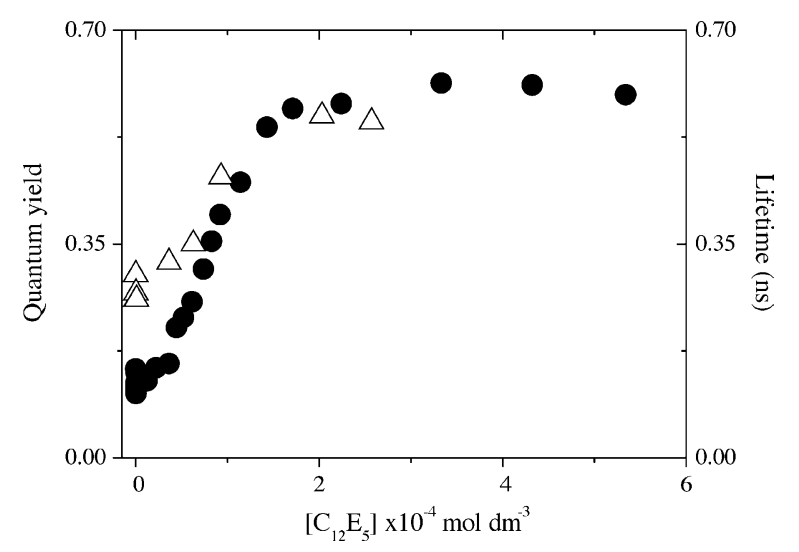

Fig. 4. Fluorescence quantum yields (circles) and lifetimes (triangles) for aqueous solutions of PBS-PFP as functions of $\mathrm{C}_{12} \mathrm{E}_{5}$ concentration.

It would seem that although there is some aggregation of polymer and surfactant, there is no specific interaction driving this.

Very different behaviour from that with either CTAB or SDS was observed with aqueous solutions of PBS-PFP in the presence of the neutral $\mathrm{C}_{12} \mathrm{E}_{5}$. A blue shift in the emission maxima (Fig. 3(c)) was seen, and was accompanied by dramatic increases in both fluorescence quantum yield and lifetime (Fig. 4). We have suggested [20] that the spectral changes, which are very similar to those observed with a phenyl-fluorene copolymer on going from thin films to isolated polymer molecules in solvents such as cyclohexane [28], are due to break up of PBS-PFP aggregates by the solvent. It is noteworthy that these properties change in the vicinity of the cmc of $\mathrm{C}_{12} \mathrm{E}_{5}\left(5 \pm 2 \times 10^{-5} \mathrm{~mol} \mathrm{dm}^{-3}\right.$ [31]). As will be discussed in Section 3.4, we believe that this is due to the polymer becoming incorporated into long, cylindrical surfactant micelles.

\subsection{Electrical conductivity of solutions}

Since PBS-PFP is a polyelectrolyte, electrical conductivity measurements are likely to be informative. We have studied the effect of the PPS-PFP concentration on the electrical conductivity in aqueous solution. The molar conductivity $(\Lambda)$ was calculated using

$\Lambda=\left(\kappa-\kappa_{0}\right) /(c \times 1000)$

where $\kappa$ and $\kappa_{0}$ are the electrolytic conductivities of solution and $c$ is the polymer concentration $\left(1.577 \times 10^{-6}\right.$ to $\left.1.495 \times 10^{-6} \mathrm{~mol} \mathrm{dm}^{-3}\right)$. The experimental electrolytic con- ductivity was around $3.5 \times 10^{-5} \mathrm{~S} \mathrm{~m}^{-1}$ and the molar conductivity depends on the square root of concentration, in agreement with the Kohlrausch equation [25]

$\Lambda=\Lambda^{0}-A c^{1 / 2}$

where $\quad \Lambda^{0}=0.362 \pm 0.011 \mathrm{~S} \mathrm{~m}^{2} \mathrm{~mol}^{-1}, \quad A=8.50 \pm 0.27$ $\mathrm{S} \mathrm{mol}^{-3 / 2} \mathrm{~m}^{7 / 2}$. The molar limiting conductivity $\Lambda^{0}$ is similar to that of other polyelectrolytes in aqueous solution [32].

The facts that the behaviour of aqueous solutions of PBSPFP, both with respect to the Beer-Lambert and Kohlrausch laws, are linear functions of concentration suggest that in the absence of surfactants the polymer aggregates may be considered as separate chemical entities.

Upon addition of CTAB to an aqueous solution of PBSPFP $\left(8.6 \times 10^{-6}\right.$ (moles monomer) $\left.\mathrm{dm}^{-3}\right)$, a linear increase in electrical conductivity was observed up to surfactant concentrations $1.35 \times 10^{-5} \mathrm{~mol} \mathrm{dm}^{-3}$, where a change of slope and second linear region was seen. As with the fluorescence measurements, this break occurs at surfactant concentrations much lower than the cmc. Further, it is observed at a monomer surfactant ratio of approximately 1:2. Since each monomer has two anionic groups, this corresponds roughly to charge neutralisation. Results are summarised in Table 2.

Electrical conductivity measurements were also made on aqueous solutions of PBS-PFP in the presence of SDS. In this case, very different behaviour was observed from the system with CTAB, and all the electrolytic conductivity data could be fitted to a single straight line over the surfactant concentration range $3 \times 10^{-4}$ to $5 \times 10^{-2} \mathrm{mmol} \mathrm{dm}^{-3}$. At higher concentrations, phase separation occurred. From the electrical conductivity behaviour and the fluorescence measurements, some kind of aggregation of the surfactant and polymer is occurring which reduces the free SDS concentration in solution. This may be a polymer-surfactant anionic mixed micelle.

A third type of behaviour was observed with solutions of PBS-PFP in the presence of $\mathrm{C}_{12} \mathrm{E}_{5}$. As both the surfactant and polymer concentrations were varied in these experiments, the results are shown in a three-dimensional plot. In this case, on adding $\mathrm{C}_{12} \mathrm{E}_{5}$, ionic conductivity, and hence molar conductivity, increased with surfactant concentration, with a sharp increase around $3 \times 10^{-5} \mathrm{~mol} \mathrm{dm}^{-3}$, where micellisation starts (Fig. 5). Possible reasons for the increase in conductivity on adding $\mathrm{C}_{12} \mathrm{E}_{5}$ are discussed elsewhere [20]. What is important for this discussion is that the break point in the curve is very close to the surfactant $\mathrm{cmc}$

Table 2

Changes of electrical conductivity $(\kappa=A+B c)$ of aqueous solutions of PBS-PFP upon addition of surfactants

\begin{tabular}{llllll}
\hline Surfactant & $\Delta c(\mathrm{mM})$ & $A\left(\mu \mathrm{cm}^{-1}\right)$ & $B\left(\mu \mathrm{cm}^{-1} \mathrm{M}^{-1}\right)$ & $R^{2}$ & 0.998 \\
CTAB & $3 \times 10^{-4}$ to $7.1 \times 10^{-3}$ & 1.566 & 157385 & 0.0135 \\
& $1 \times 10^{-2}$ to 0.21 & 2.716 & 72220.1 & 0.998 & 0.0028 \\
SDS & $3 \times 10^{-4}$ to $5 \times 10^{-2}$ & 1.039 & 75441.41 & 0.999 \\
& $3 \times 10^{-2}$ to 0.20 & Positive deviation to straight line & - \\
\hline
\end{tabular}




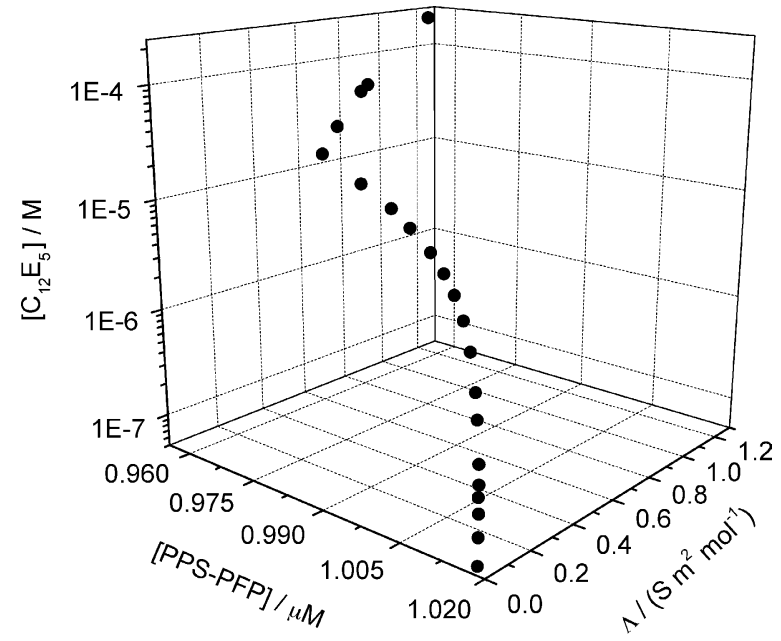

Fig. 5. Effect of $\mathrm{C}_{12} \mathrm{E}_{5}$ and polymer concentrations on the molar conductivity of PBS-PFP solutions.

$\left(5 \pm 2 \times 10^{-5} \mathrm{~mol} \mathrm{dm}^{-3}\right.$ [31]). Combining this observation with the fluorescence data gives strong evidence for a model where at surfactant concentrations above the cmc, PBS-PFP becomes incorporated as isolated, rigid-rod molecules in long cylindrical micelles. Support for this from NMR data will be given in the next section.

\subsection{NMR studies}

From ${ }^{1} \mathrm{H}$ NMR spectra, self-diffusion and proton relaxation studies, it has been shown that with increasing surfactant concentration $\mathrm{C}_{12} \mathrm{E}_{5}$ forms elongated rod-like micelles $[33,34]$. This has been confirmed by dynamic light scattering [34] and small angle neutron scattering [35]. NMR studies in such systems are expected to show considerable line broadening due to slow tumbling [36]. The ${ }^{1} \mathrm{H}$ NMR spectra were run of PBS-PFP in $\mathrm{D}_{2} \mathrm{O}$ solutions. Although in our previous report [20] we assigned sharp signals in the 6.8-7.9 ppm

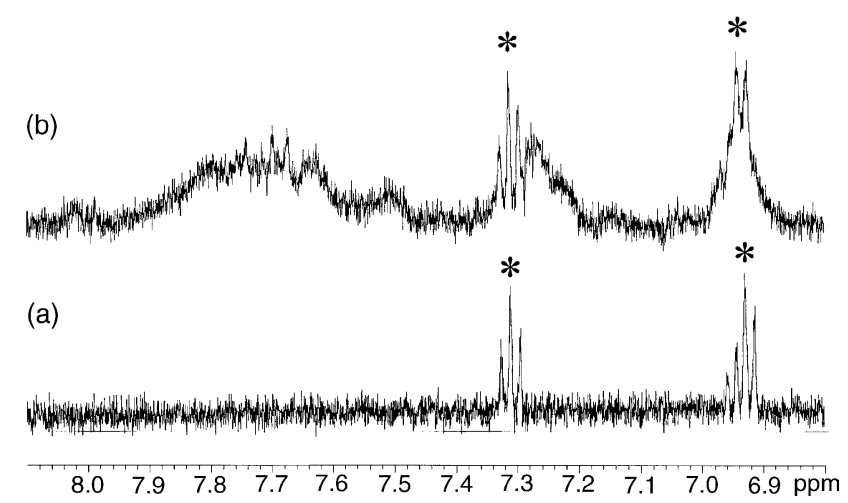

Fig. 6. NMR spectra of PBS-PFP in $\mathrm{D}_{2} \mathrm{O}$ solutions $(0.152 \mathrm{~g} / \mathrm{L})$ : (a) alone; (b) with $\mathrm{C}_{12} \mathrm{E}_{5}\left(3.4 \times 10^{-4} \mathrm{~mol} \mathrm{dm}^{-3}\right)$. Peaks marked with asterisk (*) are attributed to impurities. Reprinted with permission from H.D. Burrows, V.M.M. Lobo, J. Pina, M.L. Ramos, J. Seixas de Melo, A.J.M. Valente, M.J. Tapia, S. Pradhan, U. Scherf, Macromolecules 37 (2004) 7425 (supporting information). Copyright (2004) American Chemical Society. region (Fig. 6(a)) to the polymer, we now believe these are due to trace impurities, and that no signals attributable to PBS-PFP are observed in this region in the absence of surfactant. However, upon addition of $\mathrm{C}_{12} \mathrm{E}_{5}$ at concentrations above the surfactant cmc, broad signals due to the polymer are observed (Fig. 6(b)). These results are completely consistent with suggestions from the other techniques that PBSPFP is incorporated as isolated polymer chains in elongated, cylindrical $\mathrm{C}_{12} \mathrm{E}_{5}$ micelles. Small angle neutron scattering experiments [37] suggest that when no surfactant is present, the polymer exists as solid-like aggregates, which provides a clear explanation for the inability to observe NMR signals under these conditions.

\section{Conclusions}

The interactions of surfactants with relatively rigid-rod conjugated polymers are of interest both from theoretical and practical viewpoints. The behaviour is likely to be rather different from systems involving more flexible polymers, where pearl-necklace structures are invoked. In these studies, we have shown that the anionic PBS-PFP interacts strongly with CTAB at concentrations well below the surfactant $\mathrm{cmc}$. From both changes in the fluorescence behaviour and electrical conductivity as functions of surfactant concentration, we suggest that this is due to complexing of the cationic head group of the surfactant by the sulfonate group of the polymer through electrostatic interactions. It is probable that the alkyl chains of the surfactant become orientated close to the polymer backbone. It is hoped to test this through molecular simulations. With SDS, there is evidence of aggregation from both fluorescence and electrical conductivity measurements. Although we do not have any evidence for the type of species present, we suspect it may be some type of anionic mixed micelle. Much clearer indications of micellisation came from fluorescence, conductivity and ${ }^{1} \mathrm{H}$ NMR studies on PBS-PFP in the presence of the neutral surfactant $\mathrm{C}_{12} \mathrm{E}_{5}$. All the results strongly support a model where isolated polymer chains are incorporated in elongated, cylindrical polymer micelles. Studies in progress of the behaviour of this polymer in various solvents are consistent with it being present as isolated chains in good solvents, but forming polymer aggregates in poor ones. Within this model, the interior of $\mathrm{C}_{12} \mathrm{E}_{5}$ micelles would seem to be a favourable environment for the polymer.

\section{Acknowledgments}

We thank Professor Björn Lindman for valuable comments. We are grateful for financial support from POCTI/ FCT/FEDER, MEC/FEDER (project MAT2004-03827), MEC/CRUP (Acções Integradas), the Royal Society and BMBF (EKOS collaboration). The Universidad de Burgos is also thanked for support of a short stay by MJT in the Universidade de Coimbra. 


\section{References}

[1] R.H. Friend, R.W. Gymer, A.B. Holmes, J.H. Burroughes, R.N. Marks, C. Taliani, D.D.C. Bradley, D.A. Dos Santos, J.L. Bredas, M. Logdlund, W.R. Salaneck, Nature 397 (1999) 121.

[2] S.R. Forrest, Nature 428 (2004) 911.

[3] Y. Ohmori, M. Uchida, K. Muro, K. Yoshino, Jpn. J. Appl. Phys., Part 2: Lett. 30 (1991) L1941.

[4] U. Scherf, E.J.W. List, Adv. Mater. 14 (2002) 477.

[5] G. Lieser, M. Oda, T. Miteva, A. Meisel, H.G. Nothofer, U. Scherf, D. Neher, Macromolecules 33 (2000) 4490.

[6] M. Grell, D.D.C. Bradley, X. Long, T. Chamberlain, M. Inbasekaran, E.P. Woo, M. Soliman, Acta Polym. 49 (1998) 439.

[7] L. Chen, D. McBranch, H.-L. Wang, R. Helgerson, F. Wudl, D.G. Whitten, Proc. Natl. Acad. Sci. U.S.A. 96 (1999) 12287.

[8] O.S. Heeger, A.J. Heeger, Proc. Natl. Acad. Sci. U.S.A. 96 (1999) 12219.

[9] L. Chen, S. Xu, D. McBranch, D.G. Whitten, J. Am. Chem. Soc. $122(2000) 9302$.

[10] L. Chen, D. McBranch, R. Wang, D. Whitten, Chem. Phys. Lett. $330(2000) 27$.

[11] C. Fan, K. Plaxco, A.J. Heeger, J. Am. Chem. Soc. 124 (2002) 5642.

[12] B. Liu, B.S. Gaylord, S. Wang, G.C. Bazan, J. Am. Chem. Soc. 125 (2003) 6705.

[13] J.J. Lavigne, D.L. Broughton, J.N. Wilson, B. Erdogan, U.H.F. Bunz, Macromolecules 36 (2003) 7409.

[14] S. Wang, G.C. Bazan, Chem. Commun. (2004) 2508

[15] G. Decher, Science 277 (1997) 1232.

[16] G. Decher, M. Eckle, J. Schmitt, B. Struth, Curr. Opin. Colloid Interf. Sci. 3 (1998) 32.

[17] J.W. Baur, M.F. Rubner, J.R. Reynolds, S. Kim, Langmuir 15 (1999) 6460.

[18] J.H. Fendler, Chem. Mater. 13 (2001) 3196.

[19] H. Kobayashi, S. Kanbe, S. Seki, H. Kigchi, M. Kimura, I. Yudasaka, S. Miyashita, T. Shimoda, C.R. Towns, J.H. Burroughes, R.H. Friend, Synth. Met. 111/112 (2000) 125.
[20] H.D. Burrows, V.M.M. Lobo, J. Pina, M.L. Ramos, J. Seixas de Melo, A.J.M. Valente, M.J. Tapia, S. Pradhan, U. Scherf, Macromolecules 37 (2004) 7425.

[21] K. Holmberg, B. Jönsson, B. Kronberg, B. Lindman, Surfactants and Polymers in Aqueous Solution, 2nd ed., John Wiley, 2003.

[22] M.J. Tapia, H.D. Burrows, A.J.M. Valente, S. Pradhan, U. Scherf, V.M.M. Lobo, J. Pina, J. Seixas de Melo, J. Phys. Chem. B, submitted for publication.

[23] J. Seixas de Melo, P.F. Fernandes, J. Mol. Struct. 565 (2002) 69.

[24] G. Striker, V. Subramaniam, C.A.M. Seidel, A. Volkmer, J. Phys. Chem. B 103 (1999) 8612.

[25] R.A. Robinson, R.H. Stokes, Electrolyte Solutions, 2nd revised ed., Dover Publications Inc., New York, 2002.

[26] J. Barthel, F. Feuerlein, R. Neuder, R. Wachter, J. Sol. Chem. 9 (1980) 209.

[27] M. Bockstaller, W. Kohler, G. Wegner, D. Vlasspoulos, G. Fytas, Macromolecules 33 (2000) 3951.

[28] A. Charas, J. Morgado, J.M.G. Martinho, L. Alcácer, S.F. Lim, R.H. Friend, F. Cacialli, Polymer 44 (2003) 1843.

[29] D. Wang, J. Lal, D. Moses, G.C. Bazan, A.J. Heeger, Chem. Phys. Lett. 348 (2001) 411.

[30] P. Mukerjee, K.J. Mysels, Critical Micelle Concentration of Aqueous Surfactant Systems, National Bureau of Standards, Washington, DC, 1971.

[31] M.J.E. Carless, R.A. Challis, B.A. Mulley, J. Colloid Interf. Sci. 19 (1964) 201

[32] H. Vink, J. Chem. Soc., Faraday Trans I 77 (1981) 2439; H. Vink, J. Chem. Soc., Faraday Trans I 79 (1983) 1403.

[33] P.-G. Nilsson, H. Wennerström, B. Lindman, J. Phys. Chem. 87 (1983) 1377.

[34] T. Kato, S.-I. Anzai, S. Takano, T. Seimiya, J. Chem. Soc., Faraday Trans. 185 (1989) 2499.

[35] U. Menge, P. Lang, P.G.H. Findenegg, P. Strunz, J. Phys. Chem. B 107 (2003) 1316.

[36] J. Ulmius, H. Wennerström, J. Magn. Resonance 28 (1977) 309.

[37] M. Knaapila, L. Almásy, V.M. Garamus, S. Pradhan, C. Pearson, M.C. Petty, U. Scherf, H.D. Burrows, A.P. Monkman, Macromolecules, submitted for publication. 